\title{
Long-term result of a second or third two- stage revision total knee arthroplasty for infected total knee arthroplasty
}

\author{
Young-Hoo Kim", Jang-Won Park² and Young-Soo Jang ${ }^{1}$
}

\begin{abstract}
Background: Persistent or recurrent infection after two-stage revision total knee arthroplasty (TKA) for the treatment of an infected TKA is a dreaded complication. The purpose of the current study was to determine the ability of a second or third two-stage revision TKA to control infection, evaluate the long-term survivorship of the TKA prosthesis, and measure the functional outcome after a second or third two-stage revision TKA for reinfection.

Methods: We evaluated 63 patients (65 knees) with failed two-stage TKA treated with a second or a third twostage revision TKA. There were 25 men and 38 women (mean age, $67 \pm 10.2$ years). The mean follow-up from the time of a second two-stage TKA revision was 15.1 years (range, 10 to 19 years) and the mean follow-up from the time of a third two-stage TKA revision was 7 years (range, 5 to 10 years).

Results: Overall, infection was successfully controlled in 49 (78\%) of 65 knees after a second two-stage revision TKA was performed. In the remaining 16 knees, recurrent infection was successfully controlled in 12 knees (75\%) after a third two-stage revision TKA. Survivorship, free of implant removal for recurrent infection, was $94 \%$ at 15.1 years (95\% Cl, 91 to 100\%). Survival free of revision TKA for mechanical failure was 95\% (95\% Cl, 92 to 100\%).

Conclusions: The results of the current study suggest that a second or a third two-stage revision TKA is a reasonable option for controlling infection, relieving pain, and achieving a satisfactory level of function for patients with infected TKAs.
\end{abstract}

Keywords: Long-term result, Second two-stage revision, Third two-stage revision, Infected total knee arthroplasty, Survivorship of TKA

\section{Introduction}

The reported control rates of infection with two-stage revision total knee arthroplasty (TKA) have ranged from 72-91\% [1-9]. Recurrent or persistent infection after two-stage revision TKA for the treatment of an infected TKA is a dreaded complication. Ford et al. [1] reported that $30 \%$ of patients undergoing two-stage revision TKA had serious complications. There is relatively little literature on the treatment of reinfection following two-stage revision TKA $[6,7,9,10]$. The optimum treatment of a

\footnotetext{
* Correspondence: youngookim@ewha.ac.kr

${ }^{1}$ The Joint Replacement Center of Seoul Metropolitan, SeoNam Hospital, 20, Shinjoung ipen1ro, Yangchun-Gu, Seoul, Republic of Korea

Full list of author information is available at the end of the article
}

recurrent infection after two-stage revision TKA remains controversial and varies between patients. Treatment options include antibiotic suppression [11], open debridement [12], resection arthroplasty [13], arthrodesis, staged reimplantation of another prosthesis [14] and amputation [15]. In some patients, one may be inclined to attempt a second or third two-stage revision TKA in an effort to offer more optimal knee function to the patient. Several reports on the outcome of second or third twostage revision TKA in a small number of patients have shown that it can eradicate the infection and lead to optimal knee function $[6,7,9,10,16]$.

The purpose of the current study was to: (1) determine the ability of a second or third two-stage revision TKA

(c) The Author(s). 2021 Open Access This article is licensed under a Creative Commons Attribution 4.0 International License, which permits use, sharing, adaptation, distribution and reproduction in any medium or format, as long as you give

appropriate credit to the original author(s) and the source, provide a link to the Creative Commons licence, and indicate if changes were made. The images or other third party material in this article are included in the article's Creative Commons licence, unless indicated otherwise in a credit line to the material. If material is not included in the article's Creative Commons licence and your intended use is not permitted by statutory regulation or exceeds the permitted use, you will need to obtain permission directly from the copyright holder. To view a copy of this licence, visit http://creativecommons.org/licenses/by/4.0/. 
to control infection; and (2) evaluate the long-term survivorship of a TKA prosthesis and (3) measure the functional outcome after a second or third two-stage TKA for reinfection.

\section{Patients and methods}

We retrospectively reviewed the database of 66 patients (68 knees). These 66 patients underwent a second twostage revision TKA between January 2001 and January 2010. Of the 66 patients, 3 (4.5\%) were lost to follow-up before 1 year, leaving 63 patients (65 knees) for review. Two patients had bilateral periprosthetic joint infection of the knees and they underwent simultaneous two-stage revision TKAs. The records of 63 patients had been entered into an ongoing computerized database that was updated continuously (Fig. 1). We performed irrigation and debridement after removal of the polyethylene spacer and replaced new polyethylene spacer, with retention of prosthesis as the initial treatment in all patients. Irrigation and debridement failed in all patients undergoing irrigation and debridement, resulting in two-stage revision TKA. There were 25 men and 38 women, with a mean age of $67 \pm 10.2$ years (range, 40 to 78 years) at the time of a second revision TKA. The mean body mass index was $28.9 \pm 2.9 \mathrm{~kg} / \mathrm{m}^{2}$ (range, 22 to $38.5 \mathrm{~kg} / \mathrm{m}^{2}$ ). The study was approved by the institutional review board, and all patients provided written informed consent. The American Society of Anesthesiology (ASA) Score was 2 in 55 patients and 3 in the other 8 patients.
Patients were followed at 3 months, 1 year after a second revision TKA and then 2 or 3 years or until a recurrence of infection. The mean follow-up period was 15.1 years (range, 10 to 19 years) after a second two-stage revision TKA and the mean follow-up from time of a third twostage revision for the 12 TKAs was 7 years (range, 5 to 10 years).

Periprosthetic joint reinfection was diagnosed against the criteria of Musculoskeletal Infection Society (MSIS) [17]. Reinfection was confirmed with positive cultures through aspiration and intraoperative cultures in 59 of the 65 knees (91\%) while the other 6 knees met at least one of the 3 criteria: ESR > $20 \mathrm{~mm} / \mathrm{hr} ; \quad \mathrm{CRP}>0.5 \mathrm{mg} / \mathrm{dL}$; joint aspiration leukocyte count over 1,100 cells $/ \mu \mathrm{L}$ and neutrophil percentage greater than 64\%; evidence of purulence during the subsequent surgical intervention $[16,17]$.

Causative infective organisms included sStaphylococcus aureus in 21 knees (32\%), methicillin-resistant Sstaphylococcus aureus in 10 (15\%), Staphylococcus epidermis in 9 (14\%), Streptococcus anginosus in 7 (11\%),E nterococcus cloacae in 6 (9\%), Candida albicans in $3(5 \%)$, and Candida lusitaniae in 3 (5\%). In 6 knees (9\%), no organisms were cultured (Table 1). Sixty-two of 65 knees (95\%) had the same bacteria and 3 knees (5\%) had a different bacteria from the first two-stage revision of TKA; 60 knees (92\%) had the same bacteria and 5 knees $(8 \%)$ had a different bacteria from the second two-stage revision; 13 of 16 knees $(82 \%)$ had the same bacteria and the remaining

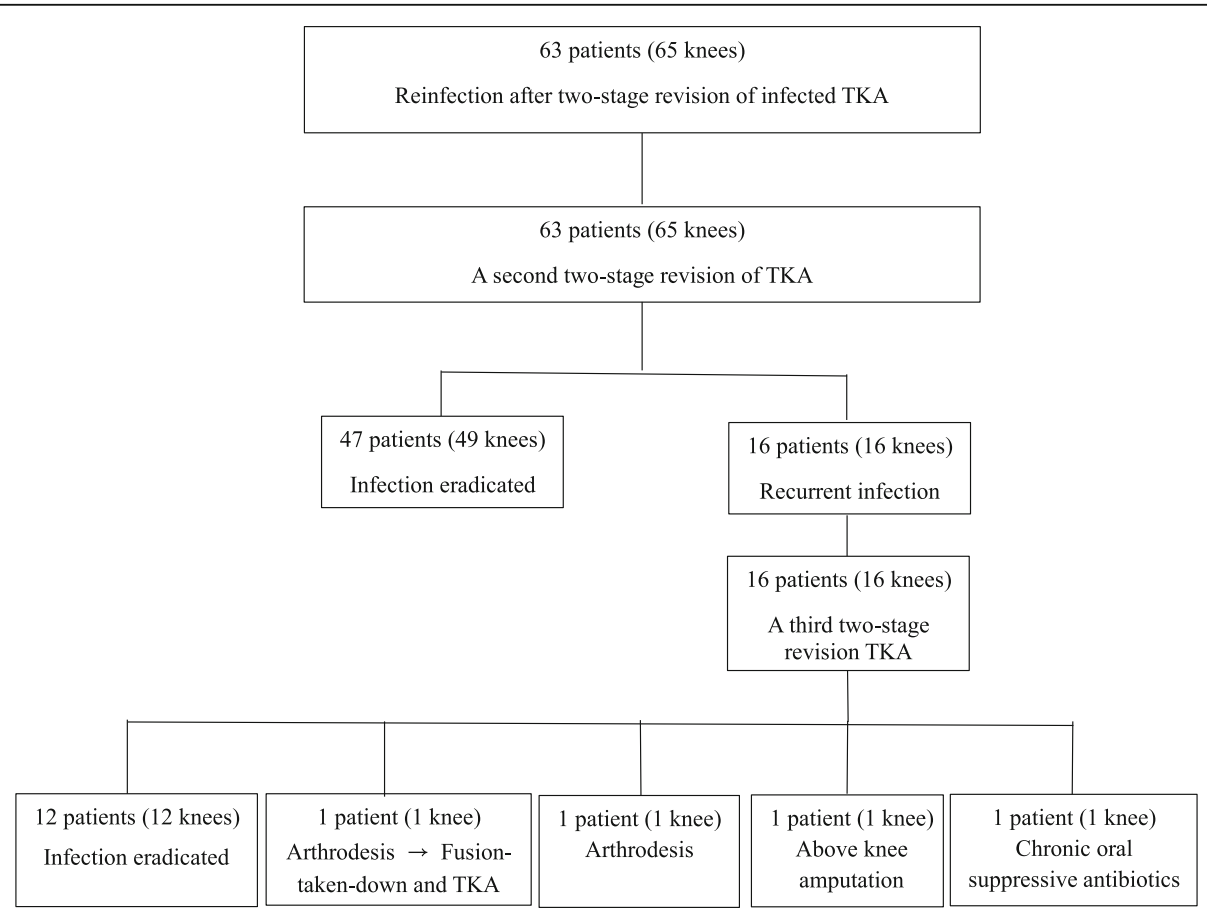

Fig. 1 Flow diagram showing the numbers of patients and knees included over the course of the follow-up period 
Table 1 Microorganism isolates and control rates of infection after a second two-stage revision TKA

\begin{tabular}{|c|c|}
\hline 1. Staphylococcus aureus & 18 of 21 knees $(86 \%)$ \\
\hline 2. Methicillin resistant Staphylococcus aureus & 6 of 10 knees $(60 \%)$ \\
\hline 3. Staphylococcus epidermidis & 8 of 9 knees (89\%) \\
\hline 4. Streptococcus anginosus & 6 of 7 knees $(86 \%)$ \\
\hline 5. Enterococcus cloacae & 4 of 6 knees $(67 \%)$ \\
\hline 6. Candida albicans & 1 of 3 knees (33\%) \\
\hline 7. Candida lusitaniae & 1 of 3 knees (33\%) \\
\hline \multirow[t]{2}{*}{ 8. No organism } & 5 of 6 knees $(83 \%)$ \\
\hline & 49 of 65 knees $(75 \%)$ \\
\hline
\end{tabular}

3 knees (18\%) had multi-organisms from the third twostage revision.

All patients underwent removal of all the well-fixed LCCK implants and, mobile bone cement spacer and debridement and placement of a tobramycin-impregnated (1.2 g per $40 \mathrm{~g}$ batch of bone cement) mobile cement spacer. Antibiotics were administered intravenously for 6 weeks. After completion of antibiotic therapy, ESR, CRP levels, total WBC count and differential in the joint aspirates and culture from the joint fluid were obtained and the patient was observed for 2 more weeks. If these tests yielded negative results and there was no clinical evidence of recurrent infection (ESR $<20 \mathrm{~mm} / \mathrm{hr}$; CRP $<$ $0.5 \mathrm{mg} / \mathrm{dl}$; and joint $\mathrm{WBC}<1100$ with $(64 \%)$, we performed a second or third two-stage revision TKA. Multiple cultures of specimens (more than 5 cultures) obtained during a second or third revision operation were performed to confirm negative culture results. The antibiotic-impregnated spacer was removed and Legacy Constrained Condylar Knee prosthesis (LCCK; Zimmer, Warsaw, Indiana) was inserted and fixed with antibioticimpregnated bone cement (1.2 g tobramycin mixed with $40 \mathrm{~g}$ of cement). For fungus infection, amphotericinimpregnated bone cement was used. After reimplantation, antibiotics were stopped at about 2 weeks by recommendation of infectious disease consultant, when the intraoperative cultures were negative, except in one patient in whom chronic oral suppressive antibiotic therapy was used.

At each follow-up, we evaluated the patients clinically and obtained radiographs of knees. Pre-revision and post-revision review data were recorded according to the systems of the Knee Society [18]. All of the knees were evaluated by one orthopedic surgeon who was not connected with the surgery, and the data were entered into a computerized record.

One of the team members evaluated the final radiographs. We defined radiographic loosening as a complete radiolucent line of $\geq 2 \mathrm{~mm}$ in width at the bone-cement or prosthesis-cement interface or a shift in position of a component on serial radiographic examination [18].

Descriptive statistics were described as the number (percentage) or mean (range). The chi-square test and Fisher exact test were used to compare binary variables. All calculations assumed 2-tailed test. The level of significance was set at $P<0.05$. All analyses were performed with SPSS, version 14.0 (SPSS Inc, Chicago, IL).

\section{Results}

Overall, 49 (75\%) of 65 knees were survived free of implant removal after a second two-stage revision TKA was performed. The remaining 16 of $65(25 \%)$ knees had a third two-stage revision TKA. At the time of a third two-stage revision TKA, femoral and tibial augmented metallic blocks were used in all of these 16 knees. None of 16 knees required a rotating hinge knee prosthesis. Twelve of 16 knees (75\%) undergoing a third two-stage revision TKA had negative culture (Table 1). In four of the 16 knees where infection was not eradicated after a third two-stage revision TKA, one knee had an aboveknee amputation, one knee had arthrodesis followed by fusion-taken-down and TKA using an LCCK prosthesis due to intact soft tissue sleeves one year after arthrodesis, one knee had arthrodesis, and one knee received chronic oral suppressive antibiotics because of a poor medical condition.

The knees with methicillin resistant Staphylococcus aureus or candida organisms tended to have a higher recurrence of infection compared with other organisms. The success rate for combined candida infections was 2 of 6 . This is significant when compared all other culture results ( 2 of 6 versus 47 of 59, $p<0.01$ ). Three knees required reoperation for aseptic loosening at a median time of 11.9 years (range, 8.5 to 15.8 years). These knees had negative cultures and negative pathology at the time of a second two-stage TKA. ESR and CRP were within normal the range. At the latest follow-up, all but four components were fixed satisfactorily.

The survivorship rate for those knees free of implant removal for reinfection was $94 \%$ at 15.1 years (confidence intervals, 91 to 100\%). The survival free-ofrevision rate for mechanical failure was 95\% (confidence intervals, 92 to $100 \%$ ) at 15.1 years (Fig. 2).

The preoperative Knee Society knee and function scores improved significantly at final follow-up. The preoperative Knee Society knee scores improved $(P \leq 0.001)$ from a median of 50 points (range, 9 to 68 points) to a median of 88 points (range, 61 to 98 points) at the final follow-up. Preoperative functional scores improved ( $p \leq$ 0.001 ) from a median of 7 points (range, 0 to 80 points) to a median of 55 points (range, 15 to 100 points) at the final follow-up. The preoperative median range of knee motion was $66^{\circ}$ (range, $15^{\circ}$ to $125^{\circ}$ ), and the median 


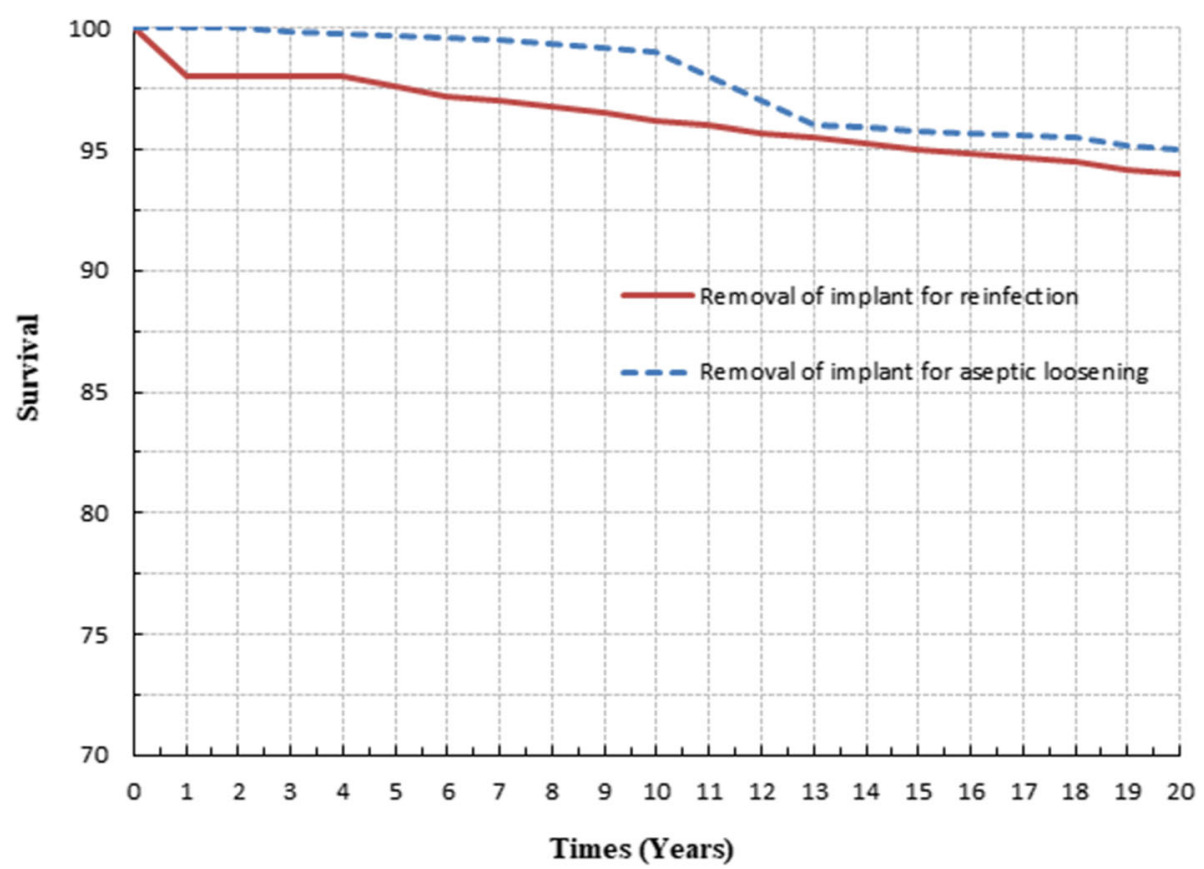

Fig. 2 Kaplan-Meier curves show survival rate of the TKA components at 15.1 years with revision TKA due to reinfection or aseptic loosening of the TKA components

range of knee motion at the final follow-up was $97^{\circ}$ (range, $30^{\circ}$ to $140^{\circ}$ ).

All but 6 knees were resurfaced patella during the second or third two-stage revision TKA. No knee suffered from periprosthetic fracture. Six of the 16 knees with a third two-stage revision TKA was not able to be resurfaced due to insufficient bone stock. The remaining 10 knees had no problem related to patella.

\section{Discussion}

The aim of this study was to determine the long-term clinical and radiographic results of the second or third two-stage revision TKA for infection using modern operative techniques and implants. The risk of failure at 15.1 years caused by recurrence of infection and mechanical reasons were approximately equal (failure rate of $5 \%$ due to mechanical future and $6 \%$ due to recurrent infection).

Patients with a previously failed two-stage revision TKA present a challenge. There is limited published data available on the second two-stage revision TKA [3, 4, 10, $16,19-21]$, with average success rate reported to be $56 \%$ (range, 4-100\%). Haleem et al. [3] reported that the survivorship free rate for implant removal for any reason was $77.3 \%$ at 10 years. Furthermore, they reported that the survivorship free rate for implant removal for reinfection was $85 \%$ at 10 years and survival free rate of revision for mechanical failure was $91 \%$ at 10 years. Backe et al. [10] reported no failure after a second two-stage reimplantation. Azzam et al. [20] reported a 78\% (14 of 18 cases) success rate after a second two-stage revision TKA. Four of 18 cases failed due to recurrent infection. Furthermore, Vadiee et al. [21] reported an overall success rate after a second two-stage revision TKA of $74 \%$ (14 of 19 cases). Stammers et al. [22] suggested that following a failed two-stage revision TKA, a second twostage revision TKA eradicated infection in 8 of 19 patients (42\%). A third two-stage revision was performed in 5 of the remaining 11 patients, eradicating infection in 3, with an average follow-up of 43 months. In the current study, irrigation and open debridement with retention of the prosthesis was tried in all knees with recurrent infection after two-stage revision TKA which doomed to failure $100 \%$. Overall success rate of salvage of the prosthesis was 78\% (49 of 65 knees) after a second two-stage revision TKA. The remaining 16 knees underwent a third two-stage revision TKA and the survival rate free of implant removal was 75\% (12 of 16 knees). Therefore, overall control rate of infection and survival rate free of implant removal, at a mean time of 15.1 years, in the current study was $94 \%$ (61 of 65 knees) after a second or a third two-stage revision TKA.

The most common microorganism identified in infection after a second two-stage revision TKA in this study was coagulase-negative Staphylococcus aureus (32\%). A similar finding has been confirmed by others $[21,22]$. The least favorable results in our study were observed in the patients who had methicillin-resistant 
Staphylococcus aureus and fungus infection. The difference in recurrence rate of infection between knees with methicillin-resistant Staphylococcus aureus and fungus and other organisms was significant $(p=0.01)$. Similar results were also observed in the aforementioned studies [9, 20, 22-25].

The strengths of this study include: (1) the relatively large number of single-surgeon patients and the longterm follow-up period; (2) the uniformity of implant designs and prosthetic fixation; and (3) the fact that this study focused on infection eradication and patient function. Our study is not without some limitations: (1) we had no comparative data on whether knee arthrodesis similarly eradicates infection or provides comparable functional results; and (2) the retrospective nature of the study may have introduced recall bias, and the review was not blinded when stratifying patient characteristics.

In conclusion, the results in the current study suggest that a second or third two-stage revision TKA is likely to result in a satisfactory outcome. In contrast, patients with methicillin-resistant Staphylococcus aureus and fungus infection tended to have a higher recurrence of infection after a second or third two-stage revision TKA.

\section{Acknowledgements}

None.

\section{Ethical statement}

The author declare that they have no financial interests or any conflict of interest. All procedures performed in studies involving human participants were in accordance with the ethical standards of the institutional and/or national research committee and with the 1964 Helsinki Declaration and its later amendments or comparable ethical standards: This article does not contain any studies with animals performed by any of the authors.

\section{Authors' contributions}

The author is the principle surgeon in all cases and the follow-up of the patients has been done under the supervision of the author. The author read and approved the final manuscript.

\section{Funding}

There is no funding from any source.

\section{Availability of data and materials}

Data were sufficient to support the study and more studies are needed in this area for final conclusion. The data are available through PubMed.

\section{Ethics approval and consent to participate}

This retrospective study was conducted after approval from the institutional review board in the authors' affiliated institution from April 2017 to December 2017. Ethical clearance was obtained from the ethical committee and approval was obtained from the institutional review board. Consent was taken from all the patients prior to inclusion into the study.

\section{Consent for publication}

I would like to confirm that the content of this manuscript has not been published elsewhere or submitted simultaneously for publication elsewhere. The manuscript has been submitted and there are no special or specific issue in the publication. All the authors give our full consent for the publication of this manuscript in the journal.

\section{Competing interests}

I would like to declare that there have been no conflicts or competing interests during the study and no issues in the journal policies. All the authors have agreed to the submission of the manuscript.

\section{Author details}

${ }^{1}$ The Joint Replacement Center of Seoul Metropolitan, SeoNam Hospital, 20, Shinjoung ipen1ro, Yangchun-Gu, Seoul, Republic of Korea. ${ }^{2}$ The Joint Replacement Center of Ewha, Womans University Seoul Hospital, Seoul, Republic of Korea.

Received: 22 June 2020 Accepted: 14 December 2020

Published online: 12 January 2021

\section{References}

1. Ford A, Holzmeister A, Rees H, Belich P. Characterization of outcomes of 2stage exchange arthroplasty in the treatment of prosthetic joint infections. J Arthroplasty. 2018;33(7 Suppl):224-27.

2. Goldman RT, Scuderi GR, Insall JN. Two-stage reimplantation for infected total knee replacement. Clin Orthop Relat Res. 1996;331:118-24.

3. Haleem AA, Berry DJ, Hanssen AD. Mid-term to long-term followup of twostage reimplantation for infected total knee arthroplasty. Clin Orthop Relat Res. 2004;428:35-9.

4. Hirakawa K, Stulberg BN, Wilde AH, Bauer TW, Secic M. Results of 2stage reimplantation for infected total knee arthroplasty. J Arthroplasty. 1998;13(1):22-8.

5. Kilgus DJ, Howe DJ, Strang A. Results of periprosthetic hip and knee infections caused by resistant bacteria. Clin Orthop Relat Res. 2002;404: 116-24.

6. Mont MA, Waldman BJ, Hungerford DS. Evaluation of preoperative cultures before second-stage reimplantation of a total knee prosthesis complicated by infection. A comparison group study. J Bone Joint Surg Am. 2000;82(11): 1552-7.

7. Wasielewski RC, Barden RM, Rosenberg AG. Results of different surgical procedures on total knee arthroplasty infections. J Arthroplasty. 1996;11(8): 931-8.

8. Windsor RE, Insall JN, Urs WK, Miller DV, Brause BD. Two-stage reimplantation for the salvage of total knee arthroplasty complicated by infection. Further follow-up and refinement of indications. J Bone Joint Surg Am. 1990;72(2):272-8.

9. Kim Y-H, Choi Y-W, Kim J-S. Treatment based on the type of infected TKA improves infection control. Clin Orthop Relat Res. 2001;469(4): 977-84.

10. Backe HA Jr, Wolff DA, Windsor RE. Total knee replacement infection after 2stage reimplantation: results of subsequent 2-stage reimplantation. Clin Orthop Relat Res. 1996;331:125-31.

11. Marculescu CE, Berbari ER, Hanssen AD, Steckelberg JM, Osmon DR. Prosthetic joint infection diagnosed postoperatively by intraoperative culture. Clin Orthop Relat Res. 2005;439:38-42.

12. Barberan J. Management of infections of osteoarticular prosthesis. Clin Microbiol Infect. 2006;12(Suppl 3):93-101.

13. Berbari EF, Osmon DR, Duffy MC, Harmssen RN, Mandrekar JN, Hanssen AD, Steckelberg JM. Outcome of prosthetic joint infection in patients with rheumatoid arthritis: the impact of medical and surgical therapy in 200 episodes. Clin Infect Dis. 2006;42(2):216-23.

14. Burnett RS, Kelly MA, Hanssen AD, Barrack RL. Technique and timing of twostage exchange for infection in TKA. Clin Orthop Relat Res. 2007:464:164-78.

15. Zimmerli W, Ochsner PE. Management of infection associated with prosthetic joints. Infection. 2003;31(2):99-108.

16. Hanssen AD, Trousdale RT, Osmon DR. Patient outcome with reinfection following reimplantation for the infected total knee arthroplasty. Clin Orthop Relat Res. 1995;321:55-67.

17. Parvizi J, Zmistowski B, Berbari EF, Bauer TW, Springer BD. New definition for periprosthetic joint infection: from the workgroup of the Musculoskeletal infection Society. Clin Orthop Relat Res. 2011;469:2992-4.

18. Insall JN, Dorr LD, Scott RD, Scott WN. Rationale of the Knee Society clinical rating system. Clin Orthop Relat Res. 1989;248:13-4.

19. Kubista B, Hartzler RU, Wood CM, Osmon DR, Hanssen AD, Lewallen DG. Reinfection after two-stage revision for periprosthetic infection of total knee arthroplasty. Int Orthop. 2012;36(1):65-71. 
20. Azzam K, McHale K, Austin M, Purtill JJ, Parvizi J. Outcome of a second twostage reimplantation for periprosthetic knee infection. Clin Orthop Relat Res. 2009:467(7):1706-14.

21. Vadiee I, Backstein DJ. The effectiveness of repeat two-stage revision for the treatment of recalcitrant total knee arthroplasty infection. J Arthroplasty. 2019;34(2):369-74.

22. Fehring KA, Abdel MP, Ollivier M, Mabry TM, Hanssen AD. Repeat two-stage exchange arthroplasty for periprosthetic knee infection is dependent on host grade. J Bone Joint Surg Am. 2017;99(1):19-24.

23. Stammers J, Kahane S, Ranawat V, Miles J, Pollock R, Carrington RW, Briggs $T$, Skinner JA. Outcomes of infected revision knee arthroplasty managed by two-stage revision of in tertiary referral centre. Knee. 2015;22(1):56-62.

24. Kim Y-H, Kulkarni SS, Park J-W, Kim J-S, Oh H-K, Rastogi D. Comparison of infection control rates and clinical outcomes in culture-positive and culture-negative infected total knee arthroplasty. J Orthopedics. 2015; 12(Suppl 1):37-43.

25. Insall JN, Thompson FM, Brause BD. Two-stage reimplantation for the salvage of infected total knee arthroplasty. J Bone Joint Surg Am. 1983; 65(8):1087-98.

\section{Publisher's Note}

Springer Nature remains neutral with regard to jurisdictional claims in published maps and institutional affiliations.

Ready to submit your research? Choose BMC and benefit from:

- fast, convenient online submission

- thorough peer review by experienced researchers in your field

- rapid publication on acceptance

- support for research data, including large and complex data types

- gold Open Access which fosters wider collaboration and increased citations

- maximum visibility for your research: over $100 \mathrm{M}$ website views per year

At $\mathrm{BMC}$, research is always in progress.

Learn more biomedcentral.com/submissions 